Published in final edited form as:

Ann Emerg Med. 2016 June ; 67(6): 706-713.e2. doi:10.1016/j.annemergmed.2015.10.025.

\title{
Observation units as substitutes for hospitalization or home discharge
}

Saul Blecker, MD, MHS ${ }^{1,2}$, Nicholas Gavin, MD ${ }^{3}$, Hannah Park, MS ${ }^{1}$, Joseph A. Ladapo, MD, $\mathrm{PhD}^{1,2}$, and Stuart D. Katz, MD, MS ${ }^{2}$

${ }^{1}$ Department of Population Health, NYU School of Medicine, New York, NY

${ }^{2}$ Department of Medicine, NYU School of Medicine, New York, NY

${ }^{3}$ Department of Emergency Medicine, NYU School of Medicine, New York, NY

\section{Abstract}

Objective-Observation unit admissions have been increasing, a trend which will likely continue due to recent changes in reimbursement policies. The purpose of this study was to determine the effect of the availability of observation units on hospitalizations and discharges to home for emergency department (ED) patients.

Methods-We studied ED visits with a final diagnosis of chest pain in the National Hospital Ambulatory Medical Care Survey from 2007-2010. ED visits that resulted in an observation unit admission were propensity-score matched to visits at hospitals without an observation unit. We used logistic regression to develop a prediction model for hospitalization versus discharge home for matched patients seen at non-observation hospitals. The model was applied to matched observation unit patients to determine the likely alternative disposition had the observation unit not been available.

Results-There were 1,325 eligible visits that represented 5,079,154 visits in the United States. Two hundred and twenty-seven visits resulted in an observation unit admission. The predictive model for hospitalization had a c-statistic of 0.91 ; variables significantly associated with subsequent hospitalization included age, history of coronary atherosclerosis, systolic blood pressure $<115$ and administration of antianginal medications. When the model was applied to matched observation unit patients, $49.9 \%$ of these patients were categorized as discharge home likely.

Corresponding Author: Saul Blecker, MD, MHS, NYU School of Medicine, 227 E. 30th St., Room 648, New York, NY 10016, Phone: 646-501-2513; Fax: 646-501-2706.

Conflict of Interest: None

An earlier version of the abstract of the manuscript was presented at the Society for General Internal Medicine Annual Meeting on April 25, 2014.

SB conceived the study. SB, NG, and SDK designed the study. SB and HP performed statistical analysis. SB drafted the manuscript. HP, NG, JL, and SDK critically revised the manuscript for important intellectual content. SB takes responsibility for the paper as a whole.

Publisher's Disclaimer: This is a PDF file of an unedited manuscript that has been accepted for publication. As a service to our customers we are providing this early version of the manuscript. The manuscript will undergo copyediting, typesetting, and review of the resulting proof before it is published in its final citable form. Please note that during the production process errors may be discovered which could affect the content, and all legal disclaimers that apply to the journal pertain. 
Conclusions-In this study, we estimated that half of ED visits for chest pain that resulted in an observation unit admission were made by patients who may have been discharged home had the observation unit not been available. Increased availability of observation units may result in both decreased hospitalizations as well as decreased discharges to home.

Utilization of observation units has increased in recent years, ${ }^{1,2}$ a trend which is likely to continue given policy pressures to reduce hospitalizations and, in particular, short term hospitalizations. ${ }^{3}$ Observation units have been associated with significant reductions in patient related costs as well as hospital length of stay. ${ }^{4-12}$ For example, early studies of observation units for chest pain suggested that placing a patient with chest pain in an observation unit would lead to a cost savings of over $25 \%$ when compared to admission to the hospital. ${ }^{4,9,13}$ More recently, observation units have been associated with reduced costs for other conditions including infectious diseases, asthma, transient ischemic attack, and heart failure. ${ }^{5,7,8,10-12}$ However, these findings of economic benefit tend to compare the cost of observation units to the cost of hospitalization, ${ }^{4-12}$ ignoring a third, and less costly, possible disposition following ED visit: discharge to home. ${ }^{14}$

Single site studies have suggested that observation units may lead to both decreased hospitalizations and decreased home discharges from the ED over time. ${ }^{15,16}$ More broadly, increased availability of clinical interventions can increase overall utilization, even in the absence of improvements in quality; for example, the increased availabilities of hospital beds, radiologic studies and cardiac catheterization have been associated with increased utilization of these services. ${ }^{17-20}$ Similarly, a supply-induced demand created by the existence of observation units may shift patients who would otherwise have been discharged home to be admitted to an observation unit.

In order to determine the extent of this potential shift in disposition, we estimated the proportion of ED patients admitted to observation units who might otherwise have been hospitalized versus discharged to home, had they been seen at a hospital without an observation unit. We hypothesized that a large portion of these ED patients would have been discharged to home.

\section{Methods}

\section{Study Design and Setting}

We examined ED visits between 2007 and 2010 using the National Hospital Ambulatory Medical Care Survey (NHAMCS). NHAMCS is an annual, national probability sample of ambulatory visits to non-federal, short-stay hospitals in the United States and is conducted annually by the Centers for Disease Control and Prevention, National Center for Health Statistics. The survey was specifically designed to provide information on the provision and utilization of ambulatory care in the United States. ${ }^{21}$

\section{Population}

For the current study, we included ED visits that resulted in an observation unit admission and compared them to ED visits at hospitals without an observation unit that resulted in a discharge to home or hospitalization. We included ED visits of patients 18 years and older 
with a first listed ED diagnosis of chest pain defined by Agency for Healthcare Research and Quality (AHRQ) Clinical Classification Software; this definition did not include specific chest pain related diagnoses such as costochondritis or myocardial infarction. Chest pain represents the most common reason for observation unit admissions following an ED visit. ${ }^{2}$

We included two groups of patient in this study: 1) those at hospitals with an observation unit that led to an observation unit admission (the treatment group) and 2) those at hospitals without a dedicated observation unit, with a disposition of either hospitalization or discharge to home (control group). We excluded patients who received endotracheal intubation or cardiopulmonary resuscitation (CPR) and those with a disposition of death, hospital transfer, left before being seen or left against medical advice (AMA), and other or missing disposition; these likely represented less than $5 \%$ of all dispositions. ${ }^{22}$

We defined hospitals to have an observation unit if they answered yes to the question: does your ED have an observation or clinical decision unit (2007-2008) or does your ED have a physically separate observation or clinical decision unit (2009-2010). Hospitals were considered to be a non-observation unit hospital if the answer to the above questions were "no" and there were no ED visits at the hospital which resulted in a disposition of an observation unit admission. We excluded visits at hospitals with an unknown observation unit status; hospital observation unit status was considered unknown if this information was missing or if the hospital reported to not have an observation unit but cared for at least one patient who was reportedly discharged to an observation unit. The number of hospitals presented represented the sum of unique hospitals per year, as NHAMCS does not track individual hospitals across years.

Patient characteristics included the following variables: age, gender, race, ethnicity, insurance, presenting vital signs (systolic blood pressure, heart rate, oxygen saturation), comorbidities (heart failure, anemia, hypertension, diabetes, coronary atherosclerosis, cardiac dysrhythmias, chronic obstructive pulmonary disease (COPD), asthma), metropolitan area, hospital region, performance of cardiac enzymes and computed tomography (CT) scan, and administration of the following medications in the ED: aspirin, antiplatelet agents, antianginal agents, anticoagulants, and nonsteroidal anti-inflammatory drugs (NSAIDs) other than aspirin. Antiplatelet agents included clopidogrel, ticlopidine, dipyridamole, and cilostazol but not aspirin; antianginal agents included nitroglycerines and ranolazine. Comorbid conditions were based on the AHRQ Clinical Classification Software definitions. As NHAMCS recorded only three diagnosis codes and study inclusion required a primary diagnosis of chest pain, patients could have at most two comorbid conditions.

The primary outcome for patients at hospitals without an observation unit was hospitalization versus discharge home. We developed a propensity score to identify a group of patients at non-observation unit hospitals most comparable to those admitted to observation units: that is, to identify those patients most likely to have been admitted to observation had they presented to an observation unit hospital. We then developed a prediction model for hospitalization for matched patients from non-observation unit hospitals; the model was applied to observation unit patients to predict disposition had the observation unit not been available. 


\section{Statistical Analysis}

We used standardized differences to compare baseline characteristics between observation units patients and patients at non-observation unit hospitals. Propensity scores were developed using a logistic regression model to determine the likelihood of receiving the "treatment" of an observation unit disposition as compared to being treated at a nonobservation unit hospital. We included all variables listed above in the matching procedure and performed propensity matching using 5:1 matching with a maximum caliper of 0.1 . The standardized difference for each covariate was calculated to confirm the covariates were balanced between matched groups. ${ }^{23}$

Using matched patients at non-observation unit hospitals, we developed a predictive model using logistic regression for likelihood of hospitalization versus home discharge. Predictors considered for the model were the same as those used for the matching procedure, although two variables (heart failure and antiplatelet agents) were not included in the final model due to collinearity. We determined the area under the receiver operating curve (AUC) for the predictive model. Additionally, we calculated positive predictive and negative predictive values for the following categorization: "hospitalization likely" if the predicted probability of hospitalization was greater than 50\% and "discharge home likely" otherwise. We then applied this categorization to observation unit patients to determine likely disposition had an observation unit not been available. As a validity check, we determined the number of those patients who were categorized as "discharge home likely" who were ultimately hospitalized, i.e. hospitalized following the admission to the observation unit. We performed a sensitivity analysis in which we varied the cut points to define "hospitalization likely." In the sensitivity analysis, hospitalization was considered the likely disposition for observation unit patients if the predicted probability of hospitalization was above $10 \%, 25 \%, 40 \%, 50 \%, 60 \%, 75 \%$, or $90 \%$.

We performed model validation using a leave one out cross validation approach. ${ }^{24}$ In this approach, we developed the predictive model as described above with excluding one matched non-observation unit patient. The excluded patient was then categorized as hospitalization likely or discharge home likely based on model prediction. We repeated this procedure for each non-observation unit patient; i.e. a new predictive model was developed for each possible set of non-observation unit patients minus one and then that one patient was categorized as hospitalization or discharge home likely based on predictive probability of that model. Categorization was compared to true disposition for all non-observation unit patients to determine positive and negative predictive values.

With one exception, all analyses accounted for the survey design and sampling weights of the NHAMCS survey. We did not account for the sampling design in the propensity matching procedure, in accordance with suggested propensity score methods. ${ }^{25}$ Analyses were performed using Stata version 13 (College Station, Texas) and are detailed in Appendix A. 


\section{Results}

\section{Characteristics of Study Subjects}

The study included 1,325 ED visits for chest pain, representing 5,079,154 visits in the United States. Of these visits, 227 patients from 457 hospitals were admitted to an observation unit; the remaining visits were for patients who were either hospitalized or discharged to home from one of 458 hospitals without an observation unit (Figure). As compared to patients at non-observation unit hospitals, observation unit patients were older (mean age 55.8 vs. 50.3), had higher rates of hypertension (12.6\% vs. $4.2 \%)$ as well as higher rates of blood pressure over $115 \mathrm{mmHG}$, and were more likely to be at a hospital in the Midwest or in an urban location. (Table 1) Observation unit patients also received higher rates of aspirin (54.9\% vs. $31.9 \%)$ and antianginal agents $(41.5 \%$ vs. $26.3 \%)$ but lower rates of NSAIDs (5.6\% vs. 13.1\%) when compared to patients at non-observation unit hospitals. (Table 1) Of patients seen at non-observation unit hospitals, $33.6 \%$ were hospitalized and $66.4 \%$ were discharged to home.

Following the propensity matching, 223 observation unit patients were matched to 1,109 patients with replacement, representing 442 unique patients at non-observation unit hospitals. Patient characteristics were well balanced following the matching procedure. (Table 1)

\section{Main Results}

Among matched patients at non-observation hospitals, characteristics associated with increased likelihood of hospitalization included increased age, Hispanic ethnicity, coronary atherosclerosis, diabetes, anemia, and receiving antianginal therapy. Asthma, systolic blood pressure greater than 115, and receipt of NSAIDs were associated with reduced risk of hospitalization. (Table 2) Due to collinearity, we neither included heart failure nor antiplatelet agents in the final model. The AUC for the predictive model for hospitalization was 0.91 ; using a cutoff in which a predictive probability $>0.5$ suggests hospitalization was likely, the positive predictive value of the model was 0.89 and the negative predictive value of the model was 0.87 . The median (IQR) of the logit scores was $0.75(-1.8,2.7)$. In cross validation, positive predictive value was 0.88 and negative predictive value was 0.83 .

When we applied the predictive model to matched patients who were admitted to an observation unit, $50.1 \%$ of the observation unit patients were categorized as hospitalization likely, while $49.9 \%$ of these patients were categorized as discharge home likely. Of those patients categorized as discharge home likely, 9.2\% were hospitalized following admission to the observation unit although this estimate should be interpreted in the context of small sample size. In sensitivity analysis, we varied the cut point for which a predictive probability of hospitalization suggested hospitalization was likely (Table 3).

\section{Limitations}

Our findings should be interpreted in consideration of study limitations. First, patient mix, care delivery, and quality of chart abstraction at hospitals with an observation unit may have inherent differences from those at hospitals without an observation unit. Although we used 
propensity matching to account for differences in patient populations, this technique does not account for unmeasured confounders. In particular, a number of potentially important variables were unavailable in NHAMCS and thus not used in the propensity and prediction models. These variables included troponin results, electrocardiogram findings, and description of the chest pain, characteristics which are necessary for clinician decision making regarding risk of acute coronary syndrome and, ultimately, the decision for hospitalization. These missing variables could have affected our results.

Our findings are based on a small sample of patients from the NHAMCS survey. As a result, findings may not be generalizable to most patients or ED settings. Underreporting of variables have been reported in the NHAMCS dataset. ${ }^{26}$ Chest pain was defined based on standard diagnostic codes, which would not have included specific diagnoses such as costochondritis or myocardial infarction. Exclusion of such patients with lower or higher propensity for hospitalizations may have influenced our findings. Hospital characteristics such as size and teaching status were not available in the dataset. The NHAMCS survey does not specifically abstract whether patients were admitted to observation status in a location other than a dedicated unit. As a result, some of the patients in non-observation unit hospitals may have received some kind of observation services. Similarly, prior work has noted potential issues with validity of the disposition variable in NHAMCS. ${ }^{27,28}$ We were unable to determine if some patients classified as observation unit patients in NHAMCS were admitted to observation services not in a dedicated unit. We analyzed a number of factors to suggest classification was correct (Appendix B), although validation by direct chart review is not possible in this de-identified dataset. We also believe that such misclassification would likely bias towards the null hypothesis, as observation patients not in a unit may be more similar to hospitalized patients. ${ }^{29}$ Of note, aspirin administration occurred in only half of observation unit patients, a finding which we believe may partially reflect pre-hospital administration of this evidence based medicine ${ }^{30}$ (Appendix C).

Due to the cross-sectional nature of this study, we were unable to determine the effect of the opening of an observation unit on either hospitalizations or home discharges. Indeed, it is possible that the initial opening of an observation unit is likely to have a different effect on utilization than the sustained availability of a unit. Lastly, we were unable to measure the effect of observation units on clinical outcomes such as rehospitalization and mortality, nor the costs in terms of the entire health-care system. Through increasing the overall number of patients being monitored in the hospital, observation units may increase the number of myocardial infarctions diagnosed and treated in the hospital. There may be particular benefits for admitting patients from vulnerable populations to an observation unit, as they may not have access to outpatient testing. Additionally, these evaluations may be performed more efficiently in the hospital as compared to the outpatient setting. The overall effect of increased utilization on population health, as well as the impact on downstream costs to the healthcare system, warrants further investigation.

\section{Discussion}

In this study, we observed there to be nearly 900,000 ED visits that resulted in an admission to an observation unit for chest pain 2007-2010. We estimated that $50 \%$ of chest pain visits 
that led to an observation unit admission would have resulted in a discharge to home, had they occurred in a hospital that lacked an observation unit. These results were, however, sensitive to our assumption that observation unit patients were hospitalized only if their predicted probability of hospitalization was greater than 50\%. For instance, when we varied this assumption to consider that observation unit patients with as low as a $10 \%$ probability of hospitalization were considered to have been likely hospitalized in the context of no observation unit availability, we found that only a quarter of observation unit patients would have been otherwise discharged to home. These findings, which must be interpreted in context of the study limitations, suggest that a substantial number of observation unit admissions are for patients who may not need to be hospitalized.

Our findings from a national sample are consistent with previous studies. Single center studies have indicated that observation units may be used for patients who may have otherwise been discharged to home. ${ }^{15,16,31,32}$ One study demonstrated that the opening of an observation unit in a hospital reduced the number of patients discharged to home from the ED by $25 \% .{ }^{15}$ A hospital registry suggested that over $10 \%$ of patients admitted to an observation unit for chest pain were very low risk and clearly avoidable. ${ }^{32}$ In a single center clustered randomized trial of effectiveness of a chest pain observation unit in the United Kingdom, the unit was not significantly associated with reduced costs; this finding was attributed to the observation unit serving as a substitute for both hospitalizations and home discharges. ${ }^{31}$ These findings were in contrast to earlier trials, which suggested that observation units reduce expenditures but compared costs to hospitalizations without consideration of the possibility of home discharge. ${ }^{33}$

In 2010, there were approximately 1.4 million total admissions to observation services in the United States. ${ }^{34}$ Utilization of observation services, which includes treatment both within and outside of a dedicated observation unit, has substantially risen in recent years; admissions to observation services more than tripled nationally from $2001-2008^{2}$ and, among Medicare beneficiaries, increased by a third relative to hospitalizations from 2007-2009. ${ }^{1}$ The number of hospitals with a dedicated observation unit has also increased in recent years. ${ }^{35}$ Nonetheless, only about $1 / 3$ of hospitals currently have a dedicated observation unit, suggesting that there is still opportunity for significant growth in the number of these units. ${ }^{34,35}$

Recent policy changes may lead to continued increase in the number of observation units. The Centers for Medicare \& Medicaid Services (CMS) has proposed that Medicare will reimburse a hospitalization only if the hospital stay includes at least two midnights, known as the Two Midnight Rule. Hospital stays of less time will be reimbursed as observation service admissions. This rule will presumably lead to significant growth in the number of admissions to observation services, which will serve as a substitute for many inpatient hospitalizations. ${ }^{3,36}$ Additionally, other payment and policy reform, such as the readmissions reductions program and accountable care organizations, have increased pressure to reduce hospitalizations and costs, which may also create incentive to open observation units. Given this likely increase in the number of patients admitted to observation services, coupled with the potential clinical and purported financial benefits of dedicated observation units, the number of observation units will likely increase 
substantially in response to this policy change. ${ }^{34}$ The results of our study suggest that the availability of observation units may increase utilization overall.

Previous economic evaluations have suggested that increased utilization of observation units will result in substantial cost savings to society. For instance, one study estimated that expanding observation units to most hospitals would result in national cost savings of $\$ 3.1$ billion. ${ }^{37} \mathrm{~A}$ second study estimated that substituting the treatment of many current short stay hospitalizations for observation unit admissions - a change beginning to occur as a result of the Two Midnight Rule - could lead to savings of over $\$ 5$ billion per year. ${ }^{34}$ The first study assumed that two-thirds of observation unit admissions serve as substitutes for hospitalization and neither study considers the potential increased cost for an observation unit admission as compared to a discharge home. In contrast, we found that only half of observation unit stays were substitutes for hospitalization and prior studies suggest observation unit admissions are significantly more costly than home discharges. ${ }^{14}$

In conclusion, we estimated that approximately half of ED visits for chest pain that resulted in an observation unit admission were for patients who would have been discharged to home had the observation unit not been available. We believe that economic evaluations of observation units should consider cost implications related to effect on both hospitalizations and home discharges. Our results suggest that increased observation unit availability may lead to both decreased hospitalizations and decreased home discharges.

\section{Acknowledgments}

Dr. Blecker was supported in part by the Agency for Healthcare Research and Quality (AHRQ) grant K08HS23683. Dr. Ladapo was supported in part by the National Heart Lung and Blood Institute grant K23 HL116787.

We thank Keith Goldfeld, DrPh at NYU School of Medicine for reviewing an earlier version of this manuscript. Dr. Goldfeld received no compensation for this work.

\section{Appendix A: Statistical Appendix}

We performed the following procedures for our statistical analysis using Stata version 13 (College Station, Texas):

1. We created a propensity score using logistic regression to predict likelihood of being in an observation unit using the pscore command in Stata. The independent variables were all baseline characteristics listed in Table 1.

2. We matched observation unit patients to non-observation patients based on propensity score, using the psmatch 2 command. We used 5:1 matching with a maximum caliper of 0.1 .

3. We evaluated post-matching balance of covariates between matched observation unit and non-observation unit patients using the pstest command.

4. Using the matched patients at non-observation unit hospitals, we developed a predictive model for likelihood of hospitalization versus home 
discharge. We used the logistic command with the prefix svy to account for survey design. Independent variables in the model were all baseline characteristics listed in Table 1 with the exception of heart failure and antiplatelet agents; we excluded these variables as they were perfectly correlated with the outcome.

5. We used the postestimation command predict to calculate predicted probabilities for both non-observation unit patients and observation unit patients. If the predicted probability was $>0.5$, the patient was categorized as "hospitalization likely;" if the predicted probability was $<0.5$, the patient was categorized as "discharge home likely." We varied this cutpoint in sensitivity analysis.

6. We calculated positive predictive value and negative predictive value of the model for non-observation unit patients by comparing the categorization of "hospitalization likely" or "discharge home likely" to true disposition (hospitalization or home discharge) for each patient. We used the $t a b$ command with prefix $s v y$ to create a $2 \times 2$ table of predicted versus actual disposition for the subgroup of non-observation unit patients.

7.

We calculated the proportion of matched observation unit patients who were categorized as "hospitalization likely" versus "discharge home likely." We used the tab command with prefix $s v y$ for the subgroup of observation unit patients.

8. To perform model validation using a leave one out cross validation, we repeated step 4 with excluding one observation unit patient from the analysis. We then repeated step 5 to categorize this patient as "hospitalization likely" versus "discharge home likely." These two steps were repeated for all non-observation unit patients. We then used the categorization of "hospitalization likely" versus "discharge home likely" based on the leave one out procedure to calculate positive and negative predictive values as in step 6 .

\section{Appendix B: Assessment of Observation Unit Disposition}

The only true method for validation of observation unit disposition would be detailed chart review for disposition with comparison to the coded disposition in the dataset. As the dataset is deidentified, this approach would be impossible. To ensure face validity of our definition of observation unit patients, we have done the following:

1. We limited our inclusion criteria for observation unit patients to those who were in a hospital which was self-reported to have an observation unit.

2. In 2009-2010, the NHAMCS survey included the following variable:

"Length of stay in observation unit (for those patients who were admitted to observation unit and then discharged)". In our study, all patients in the non-observation group in 2009-2010 were categorized as "Not admitted to observation unit and then discharged". Of observation unit patients, 55.3\% 
had a numeric observation unit length of stay listed and $22.1 \%$ were categorized as missing for this variable. The remaining $22.6 \%$ of observation unit patients in our study were categorized as "not admitted to observation unit and then discharged;" all of these patients were hospitalized following their observation unit stay. These findings support some internal validity within the NHAMCS dataset for these patients.

3. We hypothesized that, if the disposition variable was correct, observation unit patients would receive cardiovascular medications at lower rates and NSAIDs at higher rates than hospitalized patients in the non-observation unit group. Conversely, we hypothesized that patients discharged to home in the non-observation unit group would receive the lowest rates of cardiovascular medications and the highest rates of NSAIDs. To test these hypotheses, we compared medication administration among observation unit patients, non-observation unit patients who were discharged home, and non-observation unit patients who were hospitalized. We found the following results:

\begin{tabular}{cccc}
\hline & Observation Unit Patients & Discharged Patients & Hospitalized Patients \\
\hline Aspirin & 54.9 & 21.7 & 52.1 \\
Antiplatelet & 1.3 & 0.2 & 2.9 \\
Antianginal & 41.5 & 10.8 & 57.0 \\
Anticoagulant & 5.0 & 1.0 & 11.8 \\
NSAIDs & 5.6 & 17.6 & 4.1 \\
\hline
\end{tabular}

These results were consistent with our hypotheses. The lone exception was aspirin use, which was comparable among observation unit and hospitalized patients and may be biased by pre-ED use (see Appendix C).

\section{Appendix C: Aspirin Use}

The rate of aspirin use was $54.9 \%$ of patients admitted to an observation unit; this rate appears low for a well-tolerated, guideline recommended mediation for acute coronary syndrome. We hypothesized that the rate of aspirin use may reflect that many patients with chest pain take aspirin prior to presentation to the ED. Treatment guidelines recommend that Emergency Medical Services (EMS) advise patients with acute coronary syndrome symptoms to take aspirin if not contraindicated. ${ }^{1}$ In one registry from Phoenix, AZ, over $60 \%$ of patients were found to have received aspirin prior to ED arrival; the majority of these patients were administered aspirin by EMS. ${ }^{2}$

Given this background, we hypothesized that patients who arrived in the ED via ambulance would have higher rates of pre-hospital use of aspirin and, as a result, lower rates of ED aspirin use, than patients who did not arrive by ambulance. To evaluate this, we examined rates of aspirin use among observation patients by mode of arrival. We found that $35.3 \%$ of observation patients who arrived by ambulance received aspirin in the ED, while aspirin use was $64.9 \%$ for observation patients who did not arrive by ambulance. These results suggest 
that pre-hospital use of aspirin may influence ED utilization of aspirin for patients with chest pain.

1. Berg RA, Hemphill R, Abella BS, et al. Part 5: adult basic life support: 2010 American Heart Association Guidelines for Cardiopulmonary Resuscitation and Emergency Cardiovascular Care. Circulation. 2010;122(18 Suppl 3):S685-705.

2. Quan D, LoVecchio F, Clark B, Gallagher JV, 3rd. Prehospital use of aspirin rarely is associated with adverse events. Prehosp Disaster Med. 2004;19(4):362-365.

\section{References}

1. Feng Z, Wright B, Mor V. Sharp rise in Medicare enrollees being held in hospitals for observation raises concerns about causes and consequences. Health Aff (Millwood). 2012; 31(6):1251-1259. [PubMed: 22665837]

2. Venkatesh AK, Geisler BP, Gibson Chambers JJ, Baugh CW, Bohan JS, Schuur JD. Use of observation care in US emergency departments, 2001 to 2008. PLoS One. 2011; 6(9):e24326. [PubMed: 21935398]

3. Sheehy AM, Caponi B, Gangireddy S, et al. Observation and inpatient status: clinical impact of the 2-midnight rule. J Hosp Med. 2014; 9(4):203-209. [PubMed: 24677628]

4. Farkouh ME, Smars PA, Reeder GS, et al. A clinical trial of a chest-pain observation unit for patients with unstable angina. Chest Pain Evaluation in the Emergency Room (CHEER) Investigators. N Engl J Med. 1998; 339(26):1882-1888. [PubMed: 9862943]

5. Greenberg RA, Dudley NC, Rittichier KK. A reduction in hospitalization, length of stay, and hospital charges for croup with the institution of a pediatric observation unit. Am J Emerg Med. 2006; 24(7):818-821. [PubMed: 17098104]

6. Leykum LK, Huerta V, Mortensen E. Implementation of a hospitalist-run observation unit and impact on length of stay (LOS): a brief report. J Hosp Med. 2010; 5(9):E2-5. [PubMed: 20717893]

7. McDermott MF, Murphy DG, Zalenski RJ, et al. A comparison between emergency diagnostic and treatment unit and inpatient care in the management of acute asthma. Arch Intern Med. 1997; 157(18):2055-2062. [PubMed: 9382660]

8. Nahab F, Leach G, Kingston C, et al. Impact of an emergency department observation unit transient ischemic attack protocol on length of stay and cost. J Stroke Cerebrovasc Dis. 2012; 21(8):673-678. [PubMed: 21482142]

9. Roberts RR, Zalenski RJ, Mensah EK, et al. Costs of an emergency department-based accelerated diagnostic protocol vs hospitalization in patients with chest pain: a randomized controlled trial. JAMA. 1997; 278(20):1670-1676. [PubMed: 9388086]

10. Ross MA, Compton S, Medado P, Fitzgerald M, Kilanowski P, O'Neil BJ. An emergency department diagnostic protocol for patients with transient ischemic attack: a randomized controlled trial. Ann Emerg Med. 2007; 50(2):109-119. [PubMed: 17490788]

11. Rydman RJ, Isola ML, Roberts RR, et al. Emergency Department Observation Unit versus hospital inpatient care for a chronic asthmatic population: a randomized trial of health status outcome and cost. Med Care. 1998; 36(4):599-609. [PubMed: 9544599]

12. Storrow AB, Collins SP, Lyons MS, Wagoner LE, Gibler WB, Lindsell CJ. Emergency department observation of heart failure: preliminary analysis of safety and cost. Congest Heart Fail. 2005; 11(2):68-72. [PubMed: 15860971]

13. Gomez MA, Anderson JL, Karagounis LA, Muhlestein JB, Mooers FB. An emergency departmentbased protocol for rapidly ruling out myocardial ischemia reduces hospital time and expense: results of a randomized study (ROMIO). J Am Coll Cardiol. 1996; 28(1):25-33. [PubMed: 8752791]

14. Gaspoz JM, Lee TH, Weinstein MC, et al. Cost-effectiveness of a new short-stay unit to "rule out" acute myocardial infarction in low risk patients. J Am Coll Cardiol. 1994; 24(5):1249-1259. [PubMed: 7930247] 
15. Schrock JW, Reznikova S, Weller S. The effect of an observation unit on the rate of ED admission and discharge for pyelonephritis. Am J Emerg Med. 2010; 28(6):682-688. [PubMed: 20637383]

16. Brillman JC, Tandberg D. Observation unit impact on ED admission for asthma. Am J Emerg Med. 1994; 12(1):11-14. [PubMed: 8285955]

17. Fisher ES, Wennberg JE, Stukel TA, et al. Associations among hospital capacity, utilization, and mortality of US Medicare beneficiaries, controlling for sociodemographic factors. Health Serv Res. 2000; 34(6):1351-1362. [PubMed: 10654835]

18. Nallamothu BK, Rogers MA, Chernew ME, Krumholz HM, Eagle KA, Birkmeyer JD. Opening of specialty cardiac hospitals and use of coronary revascularization in medicare beneficiaries. JAMA. 2007; 297(9):962-968. [PubMed: 17341710]

19. Wennberg D, Dickens J Jr, Soule D, et al. The relationship between the supply of cardiac catheterization laboratories, cardiologists and the use of invasive cardiac procedures in northern New England. J Health Serv Res Policy. 1997; 2(2):75-80. [PubMed: 10180368]

20. You JJ, Levinson W, Laupacis A. Attitudes of Family Physicians, Specialists and Radiologists about the Use of Computed Tomography and Magnetic Resonance Imaging in Ontario. Healthc Policy. 2009; 5(1):54-65. [PubMed: 20676251]

21. Centers for Disease Control and Prevention. [Accessed November 15, 2014] About the Ambulatory Health Care Surveys. Available at: http://www.cdc.gov/nchs/ahcd/about_ahcd.htm

22. Blecker S, Ladapo JA, Doran KM, Goldfeld KS, Katz S. Emergency department visits for heart failure and subsequent hospitalization or observation unit admission. Am Heart J. 2014; 168(6): 901-908. [PubMed: 25458654]

23. Austin PC. Comparing paired vs non-paired statistical methods of analyses when making inferences about absolute risk reductions in propensity-score matched samples. Stat Med. 2011; 30(11):1292-1301. [PubMed: 21337595]

24. James, G.; Witten, D.; Hastie, T.; Tibshirani, R. An introduction to statistical learning. Springer; 2013.

25. Zanutto EL. A comparison of propensity score and linear regression analysis of complex survey data. Journal of Data Science. 2006; 4(1):67-91.

26. McCaig LF, Burt CW. Understanding and interpreting the National Hospital Ambulatory Medical Care Survey: key questions and answers. Ann Emerg Med. 2012; 60(6):716-721 e711. [PubMed: 23083968]

27. Cooper RJ. NHAMCS: does it hold up to scrutiny? Ann Emerg Med. 2012; 60(6):722-725. [PubMed: 23178018]

28. Green SM. Congruence of disposition after emergency department intubation in the National Hospital Ambulatory Medical Care Survey. Ann Emerg Med. 2013; 61(4):423-426 e428. [PubMed: 23103322]

29. Venkatesh AK, Suter LG. Observation "services" and observation "care"--one word can mean a world of difference. Health Serv Res. 2014; 49(4):1083-1087. [PubMed: 25055717]

30. Quan D, LoVecchio F, Clark B, Gallagher JV 3rd. Prehospital use of aspirin rarely is associated with adverse events. Prehosp Disaster Med. 2004; 19(4):362-365. [PubMed: 15645632]

31. Goodacre S, Nicholl J, Dixon S, et al. Randomised controlled trial and economic evaluation of a chest pain observation unit compared with routine care. BMJ. 2004; 328(7434):254. [PubMed: 14724129]

32. Mahler SA, Burke GL, Goff DC Jr, et al. Avoidable utilization of the chest pain observation unit: evaluation of very-low-risk patients. Crit Pathw Cardiol. 2013; 12(2):59-64. [PubMed: 23680810]

33. Goodacre S, Morris F, Arnold J, Angelini K. Is a chest pain observation unit likely to be cost saving in a British hospital? Emerg Med J. 2001; 18(1):11-14. [PubMed: 11310454]

34. Ross MA, Hockenberry JM, Mutter R, Barrett M, Wheatley M, Pitts SR. Protocol-driven emergency department observation units offer savings, shorter stays, and reduced admissions. Health Aff (Millwood). 2013; 32(12):2149-2156. [PubMed: 24301399]

35. Wiler JL, Ross MA, Ginde AA. National study of emergency department observation services. Acad Emerg Med. 2011; 18(9):959-965. [PubMed: 21883638] 
36. Sheehy AM, Graf BK, Gangireddy S, Formisano R, Jacobs EA. "Observation status” for hospitalized patients: implications of a proposed Medicare rules change. JAMA Intern Med. 2013; 173(21):2004-2006. [PubMed: 23978913]

37. Baugh CW, Venkatesh AK, Hilton JA, Samuel PA, Schuur JD, Bohan JS. Making greater use of dedicated hospital observation units for many short-stay patients could save $\$ 3.1$ billion a year. Health Aff (Millwood). 2012; 31(10):2314-2323. [PubMed: 23019185] 


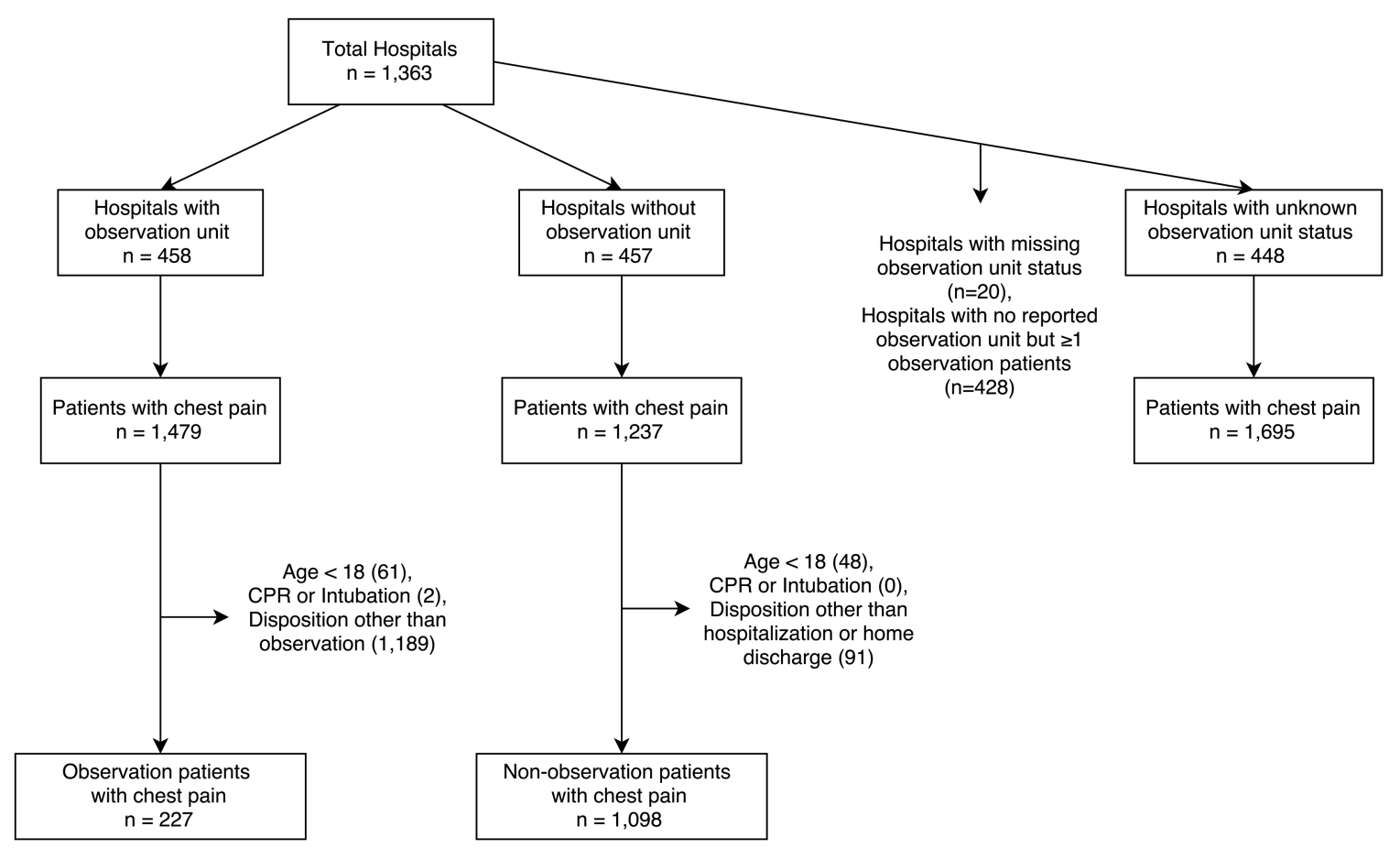

Figure.

Flowchart of hospital and patient inclusion and exclusion. The number of hospitals presented were the sum of unique hospitals per year, as NHAMCS does not track individual hospitals across years. 


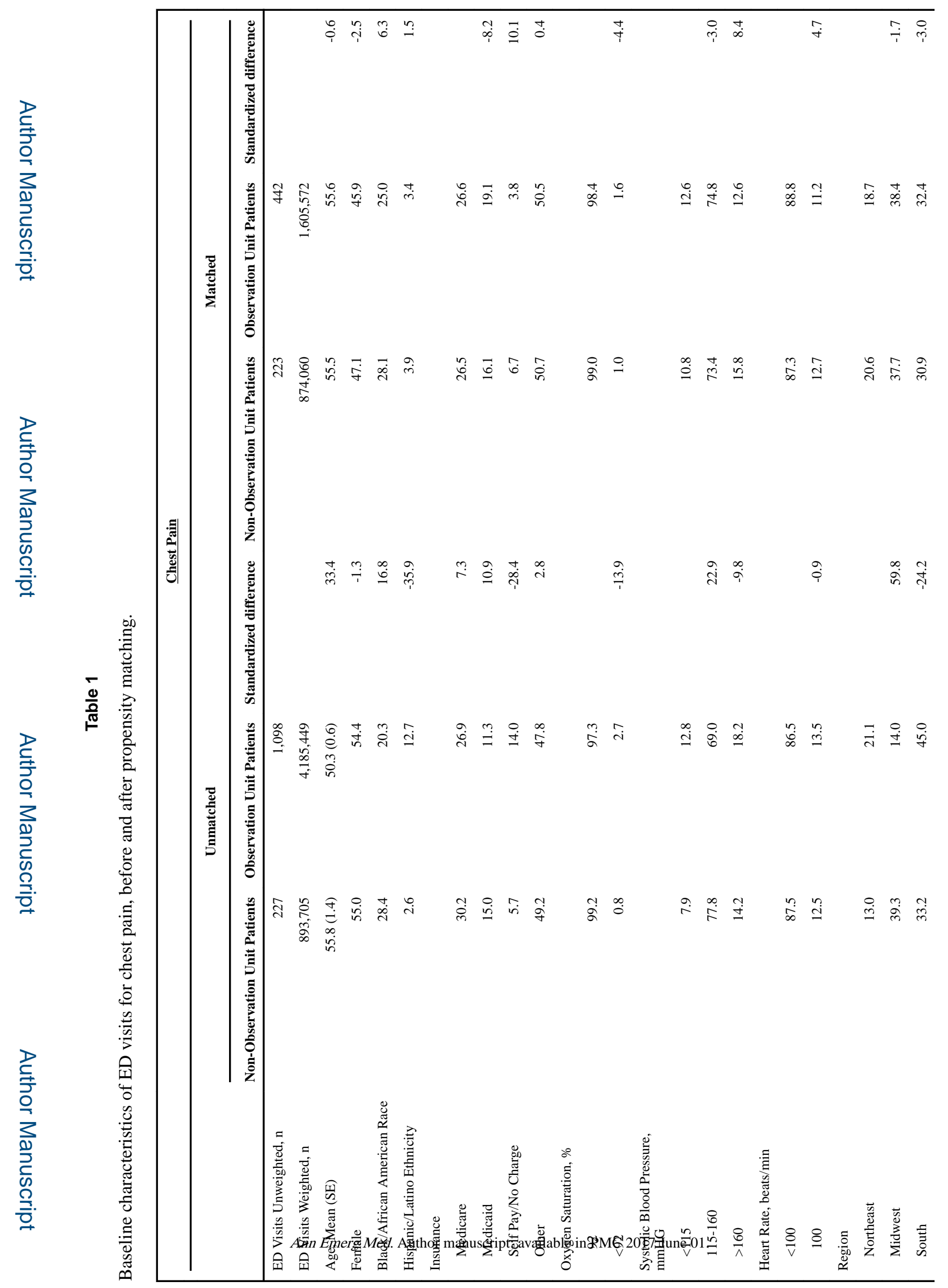




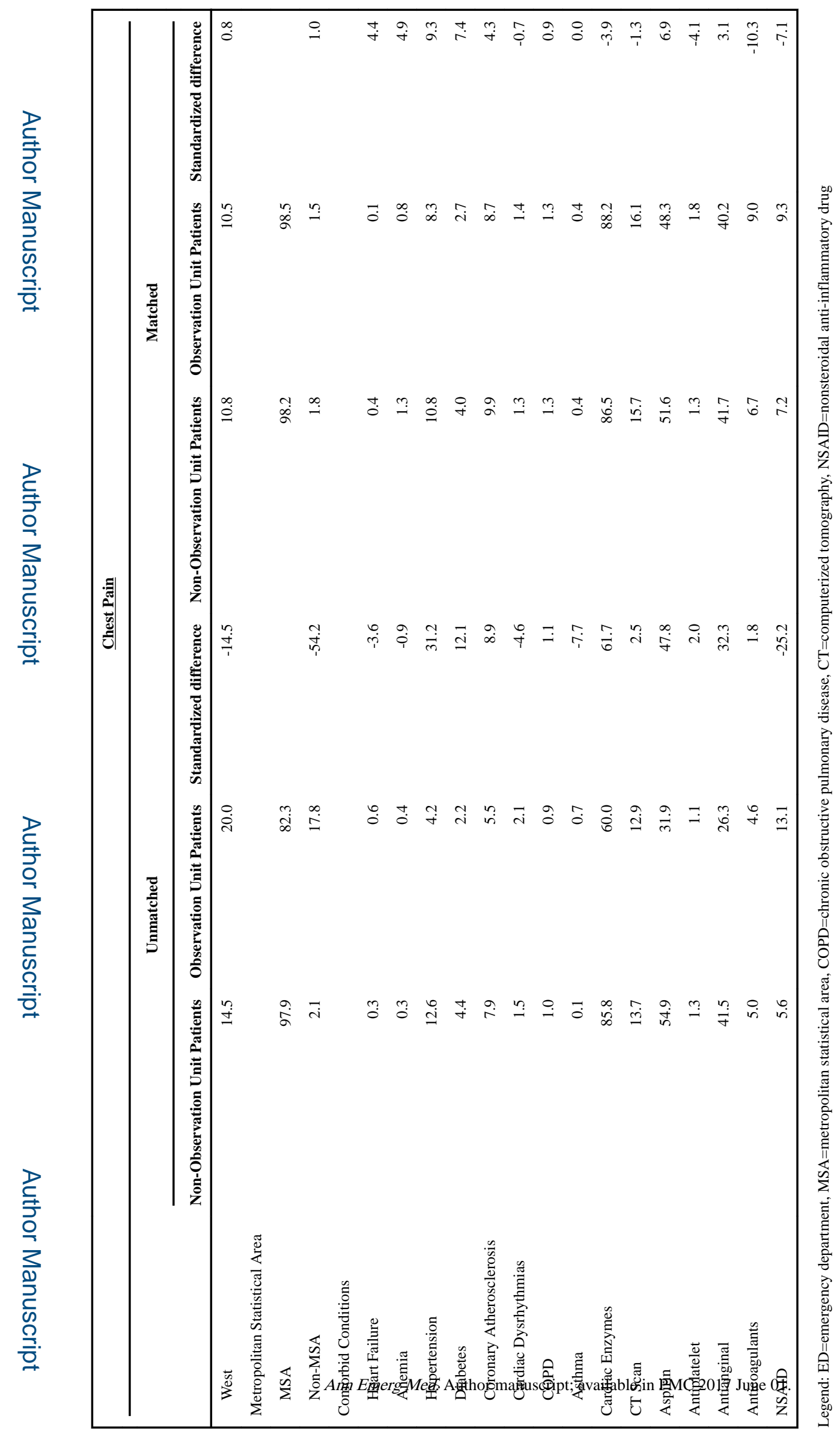




\section{Table 2}

Association of patient and emergency department (ED) characteristics with likelihood of hospitalization, among matched ED patients seen for chest pain at hospitals without observation unit.

\begin{tabular}{|c|c|}
\hline & Adjusted Odds Ratio for Hospitalization \\
\hline Age & $1.13(1.09-1.16)$ \\
\hline Female & $0.66(0.30-1.43)$ \\
\hline Black/African American Race & $2.29(0.78-6.69)$ \\
\hline Hispanic/Latino Ethnicity & $0.004(0.0001-0.14)$ \\
\hline \multicolumn{2}{|l|}{ Insurance } \\
\hline Medicare & 1 [ref] \\
\hline Medicaid & $2.36(0.47-11.78)$ \\
\hline Self Pay/No Charge & $5.02(0.68-36.84)$ \\
\hline Other & $2.57(0.89-7.37)$ \\
\hline \multicolumn{2}{|l|}{ Oxygen Saturation, $\%$} \\
\hline$\geq 92$ & 1 [ref] \\
\hline$<92$ & $2.62(0.08-80.74)$ \\
\hline \multicolumn{2}{|l|}{ Heart Rate, beats/min } \\
\hline$<100$ & 1 [ref] \\
\hline$\geq 100$ & $2.23(0.80-6.18)$ \\
\hline \multicolumn{2}{|l|}{ Systolic Blood Pressure, $\mathrm{mmHg}$} \\
\hline$<115$ & 1 [ref] \\
\hline $115-160$ & $0.14(0.03-0.54)$ \\
\hline$>160$ & $0.13(0.03-0.56)$ \\
\hline \multicolumn{2}{|l|}{ Comorbid Conditions } \\
\hline Anemia & $800.43(37.14-17251.01)$ \\
\hline Hypertension & $0.09(0.02-0.39)$ \\
\hline Diabetes & $72.21(6.49-802.72)$ \\
\hline Coronary Atherosclerosis & $11.03(1.58-76.79)$ \\
\hline Cardiac Dysrhythmias & $1.69(0.19-14.64)$ \\
\hline COPD & $0.14(0.02-0.91)$ \\
\hline Asthma & $0.12(0.02-0.70)$ \\
\hline Cardiac Enzymes & $2.70(0.80-9.11)$ \\
\hline CT Scan & $0.38(0.13-1.14)$ \\
\hline \multicolumn{2}{|l|}{ Region } \\
\hline Northeast & 1 [ref] \\
\hline Midwest & $0.94(0.35-2.49)$ \\
\hline South & $1.25(0.52-3.01)$ \\
\hline West & $0.10(0.03-0.40)$ \\
\hline Non-Metropolitan Statistical Area & $0.16(0.04-0.63)$ \\
\hline \multicolumn{2}{|l|}{ Medications Administered } \\
\hline Aspirin & $1.41(0.57-3.44)$ \\
\hline Antianginal & $43.71(19.01-100.49)$ \\
\hline
\end{tabular}




\begin{tabular}{lc}
\hline & Adjusted Odds Ratio for Hospitalization \\
\hline Anticoagulant & $2.36(0.19-29.33)$ \\
NSAIDs & $0.22(0.07-0.70)$ \\
\hline
\end{tabular}




\section{Table 3}

Sensitivity analysis in which cut point definition for categorization of hospitalization likely is varied. Patients admitted to an observation unit were considered to be likely hospitalized if their predicted probability for hospitalization was above this cut point.

\begin{tabular}{ccc}
\hline & \multicolumn{2}{c}{ Alternative Disposition for Observation Unit Patients Based on Cut Point (\%) } \\
\cline { 2 - 3 } Cut Point for Hospitalization Likely & Hospitalization Likely & Discharge Home Likely \\
\hline 0.10 & 73.7 & 26.3 \\
0.25 & 68.0 & 32.0 \\
0.40 & 58.5 & 41.5 \\
0.50 & 50.1 & 49.9 \\
0.60 & 49.1 & 50.9 \\
0.75 & 37.9 & 62.1 \\
0.90 & 28.7 & 71.3 \\
\hline
\end{tabular}

